\title{
A multi-radio, multi-hop ad-hoc radio communication network for Communications- Based Train Control (CBTC): Introducing frequency separation for train-to-trackside communication
}

Farooq, Jahanzeb; Bro, Lars; Karstensen, Rasmus Thystrup; Soler, José

Published in:

Proceedings of the IEEE Consumer Communications \& Networking Conference 2018

Link to article, DOI:

10.1109/CCNC.2018.8319169

Publication date:

2018

Document Version

Peer reviewed version

Link back to DTU Orbit

Citation (APA):

Farooq, J., Bro, L., Karstensen, R. T., \& Soler, J. (2018). A multi-radio, multi-hop ad-hoc radio communication network for Communications-Based Train Control (CBTC): Introducing frequency separation for train-totrackside communication. In Proceedings of the IEEE Consumer Communications \& Networking Conference 2018 IEEE. https://doi.org/10.1109/CCNC.2018.8319169

\section{General rights}

Copyright and moral rights for the publications made accessible in the public portal are retained by the authors and/or other copyright owners and it is a condition of accessing publications that users recognise and abide by the legal requirements associated with these rights.

- Users may download and print one copy of any publication from the public portal for the purpose of private study or research.

- You may not further distribute the material or use it for any profit-making activity or commercial gain

- You may freely distribute the URL identifying the publication in the public portal 


\title{
A multi-radio, multi-hop ad-hoc radio communication network for Communications-Based Train Control (CBTC): Introducing frequency separation for train-to-trackside communication
}

\author{
Jahanzeb Farooq ${ }^{1,2}$ Lars Bro $^{3}$, Rasmus Thystrup Karstensen ${ }^{1}$ and José Soler ${ }^{2}$ \\ ${ }^{1}$ Siemens A/S, Ballerup, Denmark \\ ${ }^{2}$ DTU Fotonik, Technical University of Denmark, Lyngby, Denmark \\ ${ }^{3}$ Nyantec UG, Berlin, Germany \\ jahanzeb.farooq@siemens.com, bro@nyantec.com, rasmus.karstensen@siemens.com,joss@ fotonik.dtu.dk
}

\begin{abstract}
Communications-Based Train Control (CBTC) is a modern signalling system that uses radio communication to transfer train control information between train and wayside. The trackside networks in these systems are mostly based on conventional infrastructure Wi-Fi (IEEE 802.11). It means a train has to continuously associate (i.e. perform handshake) with the trackside Wi-Fi Access Points (AP) as it moves, which incurs communication delays. Additionally, these APs are connected to the wayside infrastructure via optical fiber cables that incur considerable installation costs. Our earlier work presented a novel design in which trackside nodes function in ad-hoc WiFi mode, which means no handshake has to be performed with them prior to transmitting. A node upon receiving packets from a train forwards these packets to the next node, forming a chain of nodes. Following this chain, packets reach the destination. To make the design resilient against interference between the nodes, transmissions are separated on multiple frequencies, ensuring a certain separation between the transmissions. Our previous results exposed a limitation of the design. Since a train node is required to transmits on all frequencies to be able to communicate to the chain with a high probability, the frequency separation guaranteed inside the chain is not achievable in the train-to-chain communication. As a result, the train node's transmissions cause a significant amount of interference on the chain nodes. This paper proposes an extension to the design in which an additional, dedicated frequency is employed for the train-to-chain communication and presents the results from an extensive simulation study.

Index Terms-Railway signalling, CBTC, radio communica-
\end{abstract} tion, Wi-Fi, IEEE 802.11, ad-hoc, multi-radio, multi-hop

\section{INTRODUCTION}

Communications-Based Train Control (CBTC) is a widely popular modern railway signalling system that uses radio communication to transfer train control information between the train and the wayside. This results in high resolution and real-time train control information which increases the line capacity by safely reducing the distance (headway) between trains running on the same track. Despite its short range and lack of support for mobility, the IEEE 802.11 WLAN, also known as Wi-Fi, has prevailed as the radio technology of choice for CBTC systems, mainly due to its cost-effectiveness.
In these systems, hundreds of Wi-Fi Access Points (APs) are installed at the trackside to enable uninterrupted wireless connectivity. Each AP is connected to the wayside (normally a Traffic Control Center (TCC)) via optical fiber cables. Just like in an ordinary infrastructure Wi-Fi network, the train must first associate (i.e. perform handshake) to an AP to be able to transmit. However, there are a number of disadvantages of this design. Firstly, installation of cables to connect each AP to the wayside is time-consuming and incurs high costs. Secondly, the train must handover from one AP to other as the it moves. The IEEE 802.11 technology lacks the support for mobility as it was originally developed for users in stationary environments. This results in employment of complex handover algorithms in CBTC systems to enable seamless mobility. A completely seamless operation is still not feasible, leading to delays in communication as well as limitations on the maximum train speed.

In [1], we presented a novel design for an ad-hoc based radio communication network (patent pending [2]) in which there are no conventional "APs". Nodes function as ordinary Wi-Fi nodes, in an ad-hoc manner. A node broadcasts packets to any nodes within its range. As a nearby node receives the packet, it re-transmits (forwards) it, to be picked up by the next nearby node. This forms a chain of nodes. Following this chain, the packets reach the last node in the chain, which is typically connected to TCC. Thus, a train is not required to establish an association with an AP, and as a result, does not need to handover between APs. Wired links between the nodes and wayside backbone are no longer needed except for the two nodes at each end of the chain. To make the chain resilient against failures, redundancy is introduced in a way that each node forwards packets to two of its neighbors in each direction instead of one. In a conventional multi-hop adhoc network where all nodes operate on a single frequency, the capacity degrades sharply with the growing size of the network as a result of the increased interference as well as contention for the medium [3], [4], [5], [6]. Thus, to make the chain resilient against interference, in the proposed design, 
three frequencies are used in an alternating fashion such that a chain node transmits on only one frequency in each direction. In this way, a certain separation is introduced between two nodes transmitting on the same frequency.

In [1], results from an extensive simulation study were presented. While the results verified the effectiveness of the design primarily in terms of resiliency, redundancy, and scalability, they exposed two limitations of the design as well. One of them is that since the train node transmits on all three frequencies (in all directions) to maximize the probability of being able to communicate with the chain, the frequency separation inherent inside the chain is not achievable between the transmissions of the train and the chain nodes. This leads to a large amount of interference on these nodes and as a result, a significantly high packet loss. To minimize this limitation, in this paper we propose an extension (patent pending [7]) to the design in which an additional, dedicated frequency is used for train-to-chain communication.

The rest of this paper is laid out as follows. Section II presents a brief overview of CBTC systems. Section III provides an overview of the proposed design. Section IV provides an overview of the extended design together with the simulation study and the results. Section V discusses future work. Finally, Section VI concludes the paper.

\section{OVERVIEW OF CBTC SYSTEMS}

A brief overview of CBTC is presented here. For a more detailed version, refer to [8]. In CBTC, radio communication is used to exchange train control information between the train and the wayside, enabling Automatic Train Control (ATC) functions. The train regularly sends its state to the wayside over the radio connection. The state information includes the current speed, direction, and location of the train. Based on this information, the wayside ATC equipment calculates the "limit of movement authority" (LMA) information and sends it back to the train. LMA includes the maximum speed and distance the train is permitted to travel. Based on LMA, the onboard ATC equipment ensures that the train speed and the safety distance to the preceding trains conforms to the required limits. Due to this real-time communication between train and wayside, the precise location of the trains can be determined. This enables the so-called "moving block operation" that allows trains to run closer to each other. Furthermore, the number of trackside equipment—such as color light signals and track circuits-is minimized. Fig. 1 illustrates typical wayside — which includes trackside — components of a CBTC system. The wayside ATC subsystems additionally perform functions including scheduling trains and determining their destination/dwell times. These subsystems are often collectively referred to as the Traffic Control Center (TCC).

A large number of Wi-Fi APs are deployed at the trackside to guarantee that the train has a radio connection all the time. Each AP is connected (over a wired link) to the wayside components through the backbone network. A train has to continuously search for a new suitable AP (a process called scanning) and re-associate as it moves along. To assist in

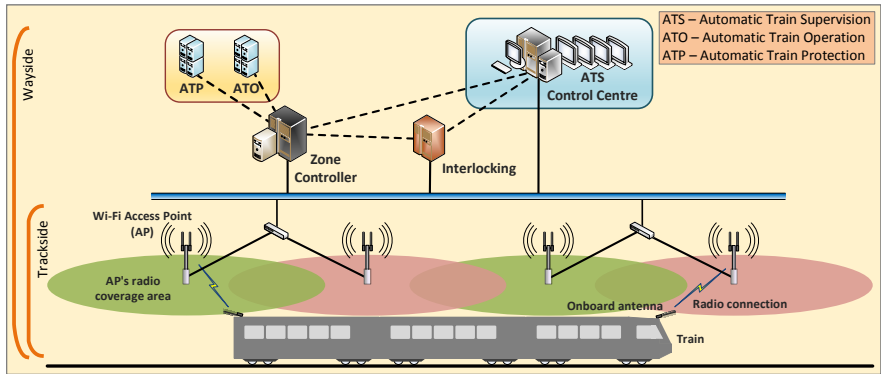

Fig. 1. CBTC wayside components

handover, APs are placed in a way that their coverage areas overlap. Fig. 1 uses the green and red colors to differentiate between the APs' coverage areas. A critical aspect of handover in CBTC is how the train smoothly switches from one AP to another, without causing interruptions in the communication. A large handover latency might result in a train failing to receive information about the minimum permitted distance to the preceding train in-time. Normally a smooth transition is ensured by equipping a train with at least two radios, one at each end, such that one of these radios stays associated to the current AP while the other switches to a new AP [8].

Normally, CBTC control messages are sent at regular, short intervals of 100-600 milliseconds. This guarantees that the wayside and the train always receive the most updated information (i.e. train state and LMA) from each other [8].

\section{Proposed Network DESIGN}

A brief description of the proposed network is presented here. For further details, refer to [1]. Fig. 2 (a) illustrates the conventional network design for CBTC trackside. In the proposed design, at its basic, a train broadcasts packets which are then picked up by a node in the chain and forwarded to its neighboring node, and so on, as illustrated in Fig. 2 (b). No AP scanning and association are thereby required.

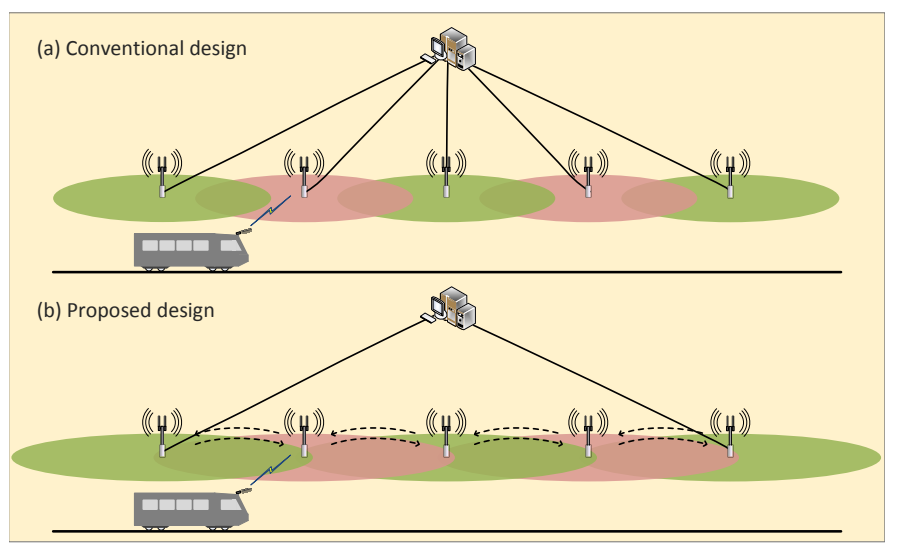

Fig. 2. CBTC trackside network: Conventional vs. proposed design

\section{A. Three frequencies and redundancy}

A conventional multi-hop ad-hoc network operating on a single frequency presents two major challenges. Firstly, as 


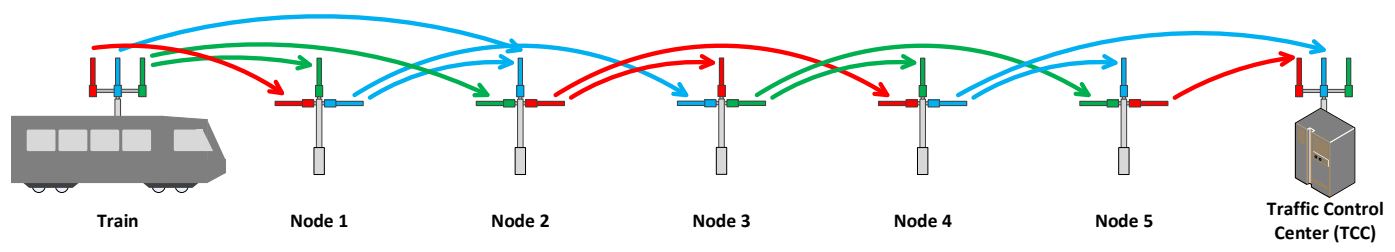

Fig. 3. A terminal node (train) transfers packets to another terminal node (TCC) over a chain of five nodes

noted above, if all nodes transmit on the same frequency, the probability of interference increases sharply. Additionally relevant is the well-known "hidden node problem" in which two nodes are in the transmission range of a common node but not in each other's range. Since they cannot hear each other, it renders the Carrier Sense Multiple Access/Collision Avoidance (CSMA/CA) mechanism used in IEEE 802.11 MAC to avoid collisions ineffective. Secondly, a single failed node practically breaks the chain.

To solve the interference problem, the proposed design uses three frequencies to ensure a certain separation between nodes transmitting on the same frequency. Each node is equipped with three radios, all on different frequencies. The two side radios use directional antennas one in each direction. Transmissions are made not only to the immediate neighbor node but also the following node. The third top radio is equipped with an omni-directional antenna and is used only for receiving. The three frequencies are then used in an alternating fashion on subsequent nodes. A predefined address included in each packet indicates the direction of the traffic flow. The three radios inside a node are connected to each other via Ethernet. As a radio receives a packet, depending on the direction of the traffic, it delivers the packet to the correct side radio (i.e. left or right) which transmits it further. Fig. 3 illustrates the mechanism where the colors red, blue and green represent three frequencies and a uni-directional traffic flow (from left to right) is depicted. As seen, the two-node transmission range solves the "single point of failure" problem and introduces redundancy as a node receives the same packet from two nodes rather than one. It further solves the "hidden node problem" by ensuring that two nodes transmitting to a third common node are always in each other's range, e.g. Node 1 right radio and Node 3 left radio transmitting to Node 2 top radio.

\section{B. Chain node vs. terminal node}

The node type discussed above is referred to as a "chain node". A second type of node is the "terminal node", which is the actual sender/receiver of these packets and is either a train or a TCC. A train sends packets to the TCC, and a TCC sends packets to one or more trains. Note that in reality, TCC is a stationary machine without any radio equipment and is connected to the end of the chain using a wired connection. Nonetheless, for simplicity, figures in this paper (e.g. Fig. 3) depict a TCC with radio equipment just like a train. In this way, it can as well be seen as a train transmitting to another train over the chain.
While a chain node transmits only on two frequencies (one in each direction), a train transmits on all three frequencies and in all directions, i.e. it uses three omni-directional antennas. This is necessary as a train shall be able to communicate to the chain regardless of its direction or orientation relative to the chain. Note that one of the directional antennas on a chain node might be facing opposite and thus might not be able to receive from the train. Thus, by transmitting on all three frequencies, it is guaranteed that a chain node with any of the three possible frequency combinations is able to receive from train on a minimum of two frequencies. Fig. 3 shows a network where a terminal node (train) transfers packets to another terminal node (TCC) over a chain of five nodes. Note how the use of three frequencies ensures a frequency separation distance of three nodes, e.g. the red frequency is used by Node 2 and Node 5 .

Regardless of the intended direction of a packet, a chain node upon receiving a packet directly from a train forwards the packet in both directions. Forwarding the packet in the backward direction ensures that the packet takes the shortest path to TCC, which might be located at either end of the chain. The following chain node (in each direction) upon receiving this packet continues to forward it in only one direction.

An inherent consequence of the redundancy in the design is the duplicate packets. Note that a node might receive multiple copies of the same packet either from the same node or from two different nodes. For example in Fig. 3, Node 1 will receive two copies of the same packet from the train. Node 2 will receive four copies of the same packet, two forwarded by Node 1 and two received directly from the train, and so on. If each node forwards the duplicate packets, they grow exponentially along the chain and congest the network. Therefore, duplicates are eliminated at each node based on a unique identifier.

\section{SimUlation STUDY}

Simulations were carried out using a discrete-event simulator [9]. Table I lists the key simulation parameters and their values used in the simulations. An inter-node distance of 600 meters has been used in all simulations, as it could be directly related to the distance currently used in the Copenhagen's Strain CBTC system based on the conventional CBTC technology. As the design requires that a node be heard by two of its neighbors, transmission power and receive sensitivity were adjusted to transmit to a distance of 1200 meters.

The proposed design relies on the assumption that the separation provided by the three-frequency design is sufficient and signals from nodes beyond that distance will not interfere. 
TABLE I

SimUlation PARAMETERS

\begin{tabular}{|l|l|}
\hline Parameters & Value \\
\hline WLAN technology & IEEE 802.11a OFDM at 54 Mbps \\
\hline Frequency channels $(\mathrm{MHz})$ & $5170,5230,5290,5735,5795$ \\
\hline Transmission power $(\mathrm{dBm})$ & 7 \\
\hline Receive sensitivity $(\mathrm{dBm})$ & -76 \\
\hline Antenna gain $(\mathrm{dBi})$ & 14 \\
\hline Antenna height $(\mathrm{m})$ & Train: 2, Chain node: 2 (side), 3 (top) \\
\hline Packet size & Payload: 512 bytes, Headers: 297 bits \\
\hline Inter-node distance $(\mathrm{m})$ & 600 \\
\hline Nodes & 20 \\
\hline Packet rate (per second) & 1000 \\
\hline Simulation time (s) & 60 \\
\hline
\end{tabular}

However, this is far from reality as minor changes in the propagation conditions have shown to dramatically increase the signal range in railway environments [8]. Our simulation model uses the simulation tool's default Free-Space Path Loss (FSPL) propagation model. The FSPL model assumes a free space between the sender and receiver and therefore does not consider signal loss that occurs due to obstacles. Thus, it enables exceptionally large signal range-or interference range-which provides the worst case scenario necessary to validate the proposed design.

\section{A. Results and discussions}

In our simulation scenarios, one or more terminal nodes located at the two ends of chain transmit packets which are then transferred to the terminal node at the other end of the chain. A network size of 20 nodes has been used, which corresponds to 12 kilometers and closely relates to the size that will be used in the actual CBTC deployments.

When discussing results, we are particularly interested in six performance indicators, namely unique packets received, duplicate packets received, total packets received, collisions, erroneous packets received, and, packets lost. Number of unique packets received serves as our key parameter as it indicates how many of the original unique packets (i.e. excluding duplicates) sent by the train are successfully transferred over the network. Note that this number for a node is essentially equivalent to the number of packets forwarded by the node. Total packets received includes duplicate packets. Erroneous packets are a result of interference between transmissions from different nodes (including collisions). These packets are discarded and do not count towards the aforementioned packets received numbers. Packets lost is the number of packets that, out of the original unique packets sent, were not received at the receiving end, for example owing to errors.

Note that while we discuss results for all nodes in the network, we are primarily interested in the results for the terminal nodes.

1) Scenario 1: Default setup: In this scenario, the performance of the default design is studied. In the first part of this scenario, one terminal node (train) transmits packets which are then transferred to the other terminal node (TCC) over the chain. A packet rate of 1000 packets per second-equivalent to $4.4 \mathrm{Mbps}$-is used.

Fig. 4 shows the results for the above mentioned six parameters against each node shown on the $\mathrm{x}$-axis. The $\mathrm{y}$-axis shows the number of packets received as a percentage of the unique packets sent. Note that with the rate of 1000 packets per second and a simulation time of 60 seconds, the number of packets sent by a single radio on a terminal node during the whole simulation run is 60,000 . Thus, a $100 \%$ unique packets received by a node implies that it received all 60,000 packets. Note that the total number of packets sent by a terminal node is thrice this number - as it sends on three radios. And ideally, the total number of packets received by a chain node in one direction is twice this number-as it receives packets from two of its preceding nodes.

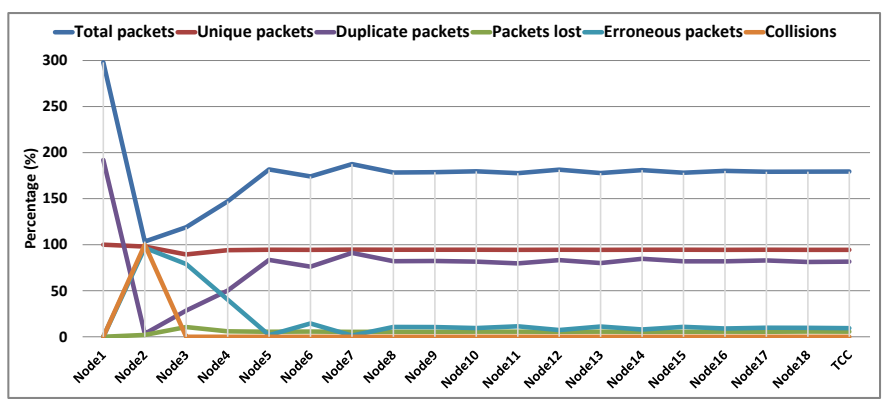

Fig. 4. Results for Scenario 1: Default design with one traffic flow

The results show that $94.37 \%$ of the unique packets (red line) were successfully transferred to TCC, i.e. a packet loss of only $5.63 \%$ over a long chain of 20 nodes. As seen, the large and stable number of duplicate packets received at each node highlights the effectiveness of the redundancy in the design. Furthermore, the frequency separation successfully minimizes interference as the number of erroneous packets is minimumexcept for the first few nodes.

The large number of erroneous packets (light blue line) seen at the nodes near the train (i.e. Nodes 2-4) is due to the above mentioned limitation of the design and results in a sharp drop in the number of total and duplicate packets received at these nodes. As discussed above, the reason is that as a train transmits on all frequencies, the inherent frequency separation guaranteed otherwise inside the chain is not fully achievable, resulting in interference on the nearby nodes. For example, at Node 2, the train's transmissions result in collisions with those of Node 1 . Note that Node 2 is the only node in this chain that is in the transmission range of two nodes transmitting on the same frequency, and thus the only to experience collisions. While Nodes 3 and 4 are outside the train's transmission range, they are still in its interference range. For example, at Node 3 , train's transmissions interfere with those of Nodes 1 and 2.

As seen in Fig. 4, the impact of this limitation is less significant due to the inherent redundancy in the design and thus only a minor drop in the number of unique packets received (red line) is seen at these nodes. However, as observed earlier 
in [1], the problem intensifies when there are failed nodes in the network. It is because in the absence of redundancy, the packet loss seen at these nodes cannot be recovered throughout the chain. Furthermore, this limitation is particularly critical due to the fact that as a train will be travelling alongside the chain, the interference currently seen on the first few nodes will be seen across each node in the chain. Multiple trains in close proximity will further worsen the situation.

In the second part of this scenario, both train and TCC transmit packets to each other. This results in two traffic flows, one in each direction, and a combined data rate of $8.8 \mathrm{Mbps}$. Fig. 5 shows the results. Note that for brevity, the figure only shows an average of the number of total and duplicate packets received at each node. In reality, a significantly higher number of packets is received at each node as it includes the packets flowing in the opposite direction as well.

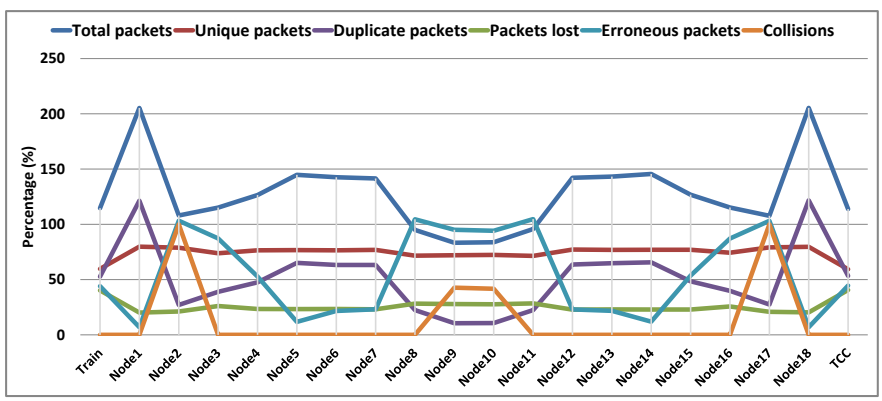

Fig. 5. Results for Scenario 1: Default design with two traffic flows

Since TCC also transmits now, in Fig. 5, an equivalent number of erroneous packets is seen at the nodes at the other end of the chain (namely Nodes 15-17) as well as a result of the interference from TCC's transmissions. While a stable number of unique packets received is maintained throughout the chain, the number is significantly lower compared to that in Fig. 4. As a result, only $59.48 \%$ of packets are successfully delivered to the terminal nodes at the each end. In other words, a packet loss of on average $40.52 \%$ is seen compared to only $5.63 \%$ in Fig. 4.

Besides the nodes at the two ends, a significant increase in the number of erroneous packets is seen at the rest of the nodes as well, resulting in a lower number of total and duplicate packets received at these nodes. This highlights the other limitation of the design reported in [1]. Specifically, it implies that despite the frequency separation distance, the chain nodes still interfere with each other as their interference range exceeds the frequency separation distance due to the favorable propagation conditions. This fact is more pronounced in Fig. 5 as the traffic is flowing in both directions and the top radio on each node faces interference from nodes on its both sides. Thus, at Nodes 5-14, on average $25.59 \%$ erroneous packets are received per flow, compared to only $8.6 \%$ in Fig. 4 . As a result, the number of total packets received at these nodes is less than $150 \%$, compared to approximately $180 \%$ in Fig. 4. This is a low number given that a node shall ideally receive two copies of the same packet, i.e. $200 \%$. Likewise, the number of duplicate packets received is less than $65 \%$, a much lower number than the ideal $100 \%$. Note that the nodes further in the middle, namely Nodes 8-11, receive the highest number of erroneous packets as these nodes have equal number of nodes at their each side to interfere with each other.

To summarize it, two limitations are identified here: (1) a train's transmissions on all frequencies cause interference on the nearby chain nodes, (2) despite the frequency separation distance, nodes beyond this distance still interfere due to their large interference range. The focus of this paper is to find a solution for the first of these limitations, which, as we observe subsequently, makes the major part of the packet loss. A solution of the second limitation has been proposed in [10].

2) Scenario 2: Separate frequency for train-to-chain communication: To minimize the interference that a train node causes on its nearby chain nodes, in this scenario, we employ a dedicated new frequency for the train-to-chain communication. Fig. 6 illustrates the mechanism where the yellow color represents the new frequency.

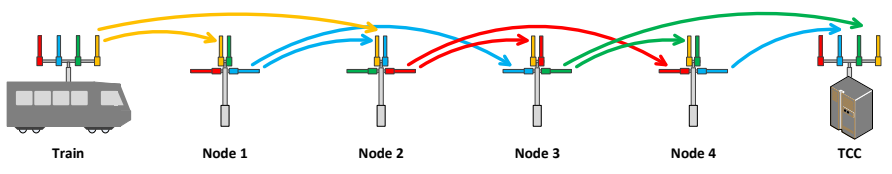

Fig. 6. Separate frequency for train-to-chain communication

Each node in this design is equipped with an additional radio that operates on this dedicated frequency. All the transmissions from train to chain are now made on this new dedicated frequency. On the other hand, a chain node, upon receiving a packet from a train, still uses the original three frequencies for forwarding the packet to the other chain nodes as in the original design. Thus, in this way, the transmissions from the train do not interfere with those from the chain nodes. Transmissions from chain-to-train are also made on the existing three frequencies as before.

Fig. 7 shows the results for the proposed extension for the one-flow scenario. The effectiveness of the new design is evident as the erroneous packets seen at Nodes 2-4 in Fig. 4 have disappeared and so has the drop in the total and duplicate packets received at these nodes. As a result, the packet loss at the TCC has dropped from $5.63 \%$ to only $0.41 \%$.

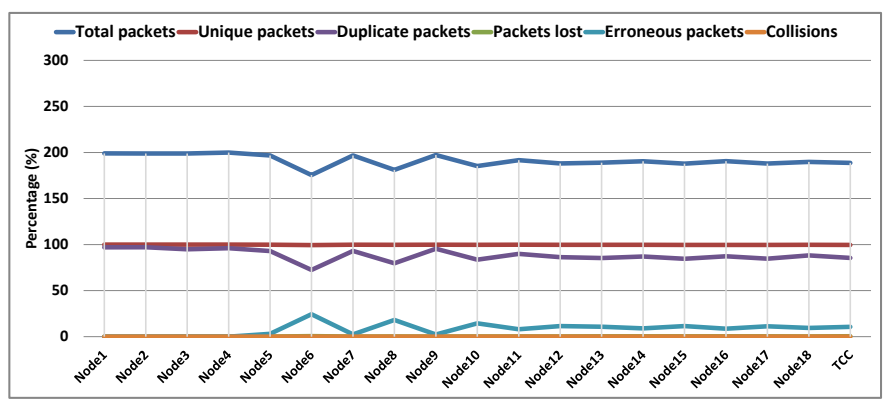

Fig. 7. Results for Scenario 2: Separate frequency for train-to-chain communication with one traffic flow 
Fig. 8 shows the results for the two-flow scenario. As expected, the erroneous packets have disappeared at both ends of the chain and the packet loss at the two terminal nodes has dropped from $40.52 \%$ seen in Fig. 5 to $17.85 \%$. This indicates that a major part of the packet loss- $22.67 \%$ out of $40.52 \%$ - was caused by the terminal nodes' transmissions. As we observe subsequently, the rest $17.85 \%$ packet loss is caused by the interference between the chain nodes. As discussed above, it is due to their interference range being larger than the frequency separation distance.

It is worth noting that in addition to employing a separate frequency, the extended design can as well be used to employ a separate radio technology for the train-to-chain communication, e.g. a combination of technologies where LTE or IEEE 802.11p is used for the train-to-chain communication and traditional Wi-Fi for the in-chain communication.

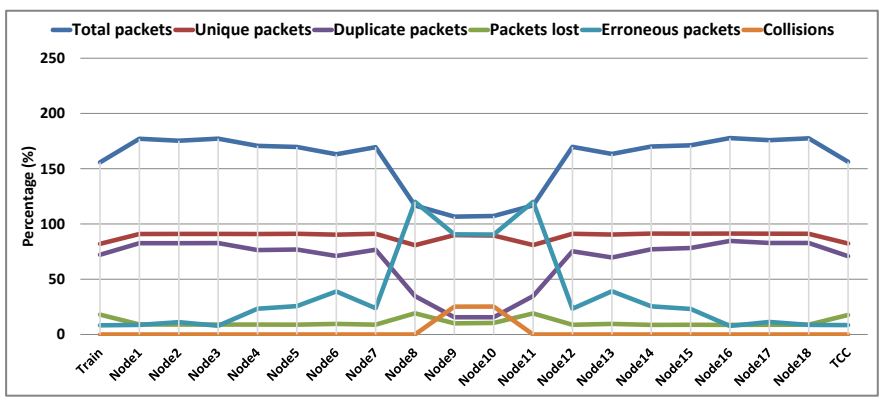

Fig. 8. Results for Scenario 2: Separate frequency for train-to-chain communication with two traffic flows

3) Scenario 3: Extended frequency separation inside the chain: To minimize the interference between the chain nodes, in this scenario, we employ an earlier extension proposed in [10] in which additional frequencies are employed by the chain nodes to extend the frequency separation distance. Fig. 9 illustrates the mechanism.

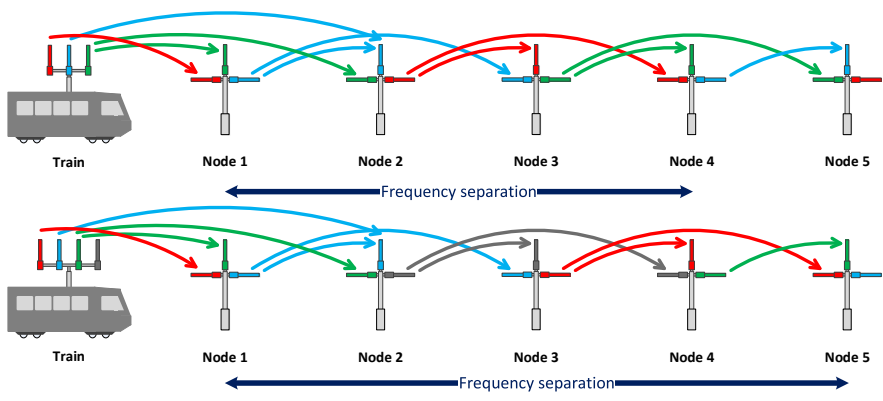

Fig. 9. Frequency separation guaranteed with three and four frequencies

The top part of Fig. 9 shows the default three-frequency design. Here, the frequency separation distance is three nodes, e.g. the blue frequency is used first by Node 1 and then by Node 4. The bottom part of the figure uses one additional frequency (black). Note that a chain node still uses three frequencies. The only difference is that now the subsequent nodes use a different set of three frequencies instead of the same frequencies in an alternating fashion. On the other hand, the train node is preferably required to be equipped with one additional radio because, as discussed in III-B, the train must use all frequencies to maximize the probability of being able to communicate to the chain. Note that it is normal to employ various-e.g. up to four-radios per train in the conventional CBTC systems in order to ensure high availability [8]. Thus, this additional radio on the train does not necessarily increase the system's cost. As seen, employing an additional frequency extends the frequency separation distance from three nodes to four nodes as the blue frequency is now repeated at Node 5 instead of Node 4.

Fig. 10 illustrates the mechanism when the two extensions are combined, i.e. the yellow frequency is used for trainto-chain communication and the black frequency is used for communication between chain nodes in addition to the original three frequencies.

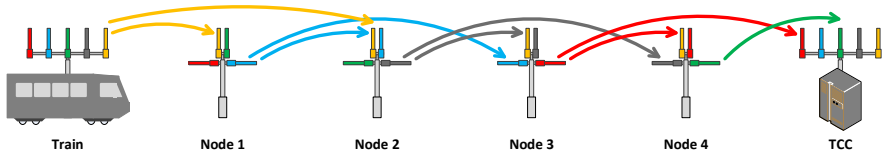

Fig. 10. Separate frequency for train-to-chain communication combined with extended frequency separation

The results for the design with the combined extensions are presented in Fig. 11. As seen when compared to Fig. 8, the extended frequency separation has resulted in a lowered number of erroneous packets at Nodes 5-14. Thus, a significant drop is seen in the number of packets lost (green line) at each node and consequently, a rise in the number of unique packets received. As a result, the packet loss at the terminal nodes has dropped from $17.85 \%$ seen in Fig. 8 to only $1.1 \%$.

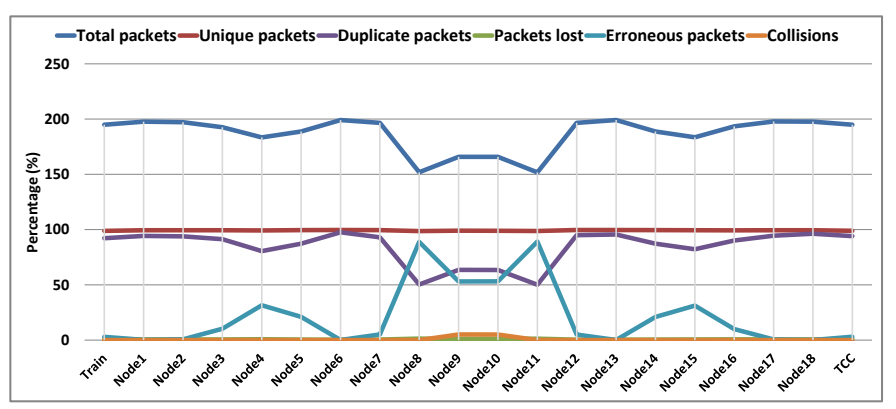

Fig. 11. Results for Scenario 3: Separate frequency for train-to-chain communication combined with extended frequency separation

The results indicate that the interference caused by the terminal nodes at their nearby nodes is a greater contributor to the total packet loss. Specifically, out of the $40.52 \%$ packet loss seen in the default scenario (Fig. 5), 22.67\% was introduced by the terminal nodes and $16.89 \%$ by the interference between the chain nodes.

The results demonstrate the effectiveness of the design as it allows to optimize frequency separation by employing 
additional frequencies, both for train-to-chain and in-chain communication.

\section{FutURE WORK}

Additional solutions to minimize the interference caused by a terminal node's transmissions and an interference range larger than the frequency separation distance, e.g. by modifying the number of radios on the train, transmission power, receive sensitivity, and modulation type, will be investigated in future. Afterward, scenarios with mobility and a greater number of trains and tracks will be considered.

\section{CONCLUSIONS}

This paper extends the previously presented design of an ad-hoc based trackside radio communication network for train to trackside communication in CBTC. A node in this design functions in ad-hoc mode, receiving broadcast packets and forwarding to its neighbors, thus forming a chain of nodes. Thus, in contrast to conventional infrastructure $\mathrm{Wi}-\mathrm{Fi}$, the train does not have to perform a handshake with the nodes as it moves and the costly optical fiber cables connecting the nodes are no more needed. The design offers resiliency against interference by employing multiple frequencies. Nonetheless, our previous results showed a significant amount of packet loss due to the interference caused by the transmissions from the train nodes, which, as per the design, are required to transmit on all frequencies and thus undermine the frequency separation guaranteed inside the chain. This paper extends the design by employing an additional, dedicated frequency to introduce frequency separation for the train-to-chain communication. The results show that the proposed extension successfully eliminates the interference caused by the train and as a result, a significantly large numbers of packets can be transferred across large networks with only limited packet loss.

\section{ACKNOWLEDGMENTS}

The authors would like to thank Simon Staudt, Kasper Tipsmark Therkildsen and Kell Quist Jensen for their support in carrying out this work.

\section{REFERENCES}

[1] J. Farooq, L. Bro, R. T. Karstensen, and J. Soler, "A multi-radio, multi-hop ad-hoc radio communication network for CommunicationsBased Train Control (CBTC)," in Proc. IEEE 86th Vehicular Technology Conference (VTC 2017-Fall), accepted for publication.

[2] Siemens AG, "Ad-hoc kommunikationsnetzwerk," Patent Application 10 2017203 040.2, feb 24, 2017.

[3] S. Xu and T. Saadawi, "Does the IEEE 802.11 MAC protocol work well in multihop wireless ad hoc networks?" IEEE Commun. Mag., vol. 39, no. 6, pp. 130-137, jun 2001.

[4] J. Li, C. Blake, D. S. De Couto, H. I. Lee, and R. Morris, "Capacity of ad hoc wireless networks," in Proc. 7th Annual International Conference on Mobile Computing and Networking (MobiCom '01). New York, NY, USA: ACM, 2001, pp. 61-69.

[5] K. Xu, M. Gerla, and S. Bae, "How effective is the IEEE 802.11 RTS/CTS handshake in ad hoc networks," in Proc. IEEE Global Telecommunications Conference (GLOBECOM 'O2), nov 2002.

[6] F. Ye, S. Yi, and B. Sikdar, "Improving spatial reuse of IEEE 802.11 based ad hoc networks," in Proc. IEEE Global Telecommunications Conference (GLOBECOM '03), dec 2003.
[7] Siemens AG, "Kommunikationsnetzwerk und verfahren zum betrieb eines kommunikationsnetzwerkes," Patent Application 102017210 668.9, jun 23, 2017.

[8] J. Farooq and J. Soler, "Radio communication for communications-based train control (CBTC): A tutorial and survey," IEEE Commun. Surveys Tuts., vol. PP, no. 99, pp. 1-1, 2017.

[9] "OPNET Modeler," Riverbed Technology, https://www.riverbed.com/dk/products/steelcentral/opnet.html.

[10] J. Farooq, L. Bro, R. T. Karstensen, and J. Soler, "A multi-radio, multi-hop ad-hoc radio communication network for CommunicationsBased Train Control (CBTC) with optimized frequency separation," in Proc. The IEEE 87th Vehicular Technology Conference (IEEE VTC2018Spring), submitted for publication. 